\title{
Generalized differential equations
}

\section{satisfying Carathéodory type conditions}

\section{J.L. Davy}

This thesis studies generalized differential equations of the form $x^{\prime} \in F(t, x)$ which satisfy Carathéodory type conditions. These consist of a measurability condition in $t$, a continuity condition in $x$, and a boundedness condition.

The thesis commences with an introduction and a chapter on set valued functions. This is designed to make the thesis accessible to anyone with a knowledge of real analysis.

When $F(t, x)$ is always a convex set, we prove that the solution set is connected and that points on the boundary of the attainable set are peripherally attainable. This is done without the additional assumption of continuity in $x$ which was required in previous results.

In the nonconvex case Filippov has proved that solutions always exist if $F(t, x)$ is continuous jointly in $t$ and $x$. We extend this result to equations satisfying Carathéodory type conditions.

Quesitrajectories are limits of approximate solutions. We study the existence of quasitrajectories and the equivalence of quasitrajectories and solutions of the equation $x^{\prime} \epsilon \operatorname{coF}(t, x)$.

When $F(t, x)$ is Lipschitz continuous in $x$, we show that the attainable set maps open sets into open sets and that every point on the boundary of the attainable set is attainable only by solutions which lie on the boundary of the attainable set. If $F(t, x)$ is also convex we prove that the solution set is a continuous function of the initial conditions.

Received 8 March 1974. Thesis submitted to the Australian National University, March 1974. Degree approved, July 1974. Supervisor: Mr W.A. Coppel. 
If every solution $x(t)$, which lies always on the boundary of the attainable set, has its derivative on the boundary of $F(t, x(t))$ almost everywhere, the equation $x^{\prime} \in F(t, x)$ is said to have the bang-bang property. If $F(t, x)$ is always a convex set and is continuous jointly in $t$ and $x$, the equation $x^{\prime} \in F(t, x)$ has the bang-bang property. We study under what type of Carathéodory conditions this result can be true.

In this thesis we try to prove our results under the most general Carathéodory type conditions, or to produce counterexamples to show that this cannot be done.

The thesis concludes with a bibliography of over one hundred works on generalized differential equations.

Part of this thesis has already been published in [1].

\section{Reference}

[1] J.L. Davy, "Properties of the solution set of a generalized differential equation", Bull. Austral. Math. Soc. 6 (1972), 379-398. 